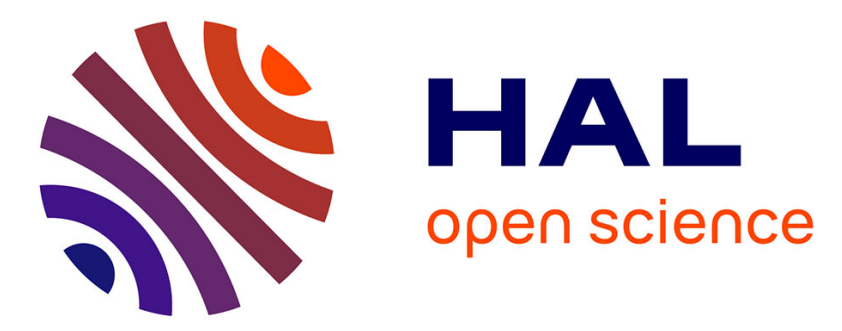

\title{
The analysis of vehicle's in-flight behaviour using quasi-LPV and nonlinear models
}

Dawid Machala, Simona Dobre, Marie Albisser, Marion Gilson, Floriane Collin

\section{To cite this version:}

Dawid Machala, Simona Dobre, Marie Albisser, Marion Gilson, Floriane Collin. The analysis of vehicle's in-flight behaviour using quasi-LPV and nonlinear models. AIAA Scitech 2019 Forum, Jan 2019, San Diego, CA, United States. pp.1321, 10.2514/6.2019-1321 . hal-02020682

\section{HAL Id: hal-02020682 https://hal.science/hal-02020682}

Submitted on 6 Sep 2019

HAL is a multi-disciplinary open access archive for the deposit and dissemination of scientific research documents, whether they are published or not. The documents may come from teaching and research institutions in France or abroad, or from public or private research centers.
L'archive ouverte pluridisciplinaire HAL, est destinée au dépôt et à la diffusion de documents scientifiques de niveau recherche, publiés ou non, émanant des établissements d'enseignement et de recherche français ou étrangers, des laboratoires publics ou privés. 


\title{
The Analysis of Vehicle's In-Flight Behaviour Using Quasi-LPV and Nonlinear Models
}

\author{
Dawid Machala* \\ French-German Research Institute of Saint Louis (ISL), 5 Rue du Général Cassagnou, 68301, France \\ Université de Lorraine, CNRS, CRAN, F-54000 Nancy, France \\ Simona Dobre ${ }^{\dagger}$ and Marie Albisser ${ }^{\dagger}$ \\ French-German Research Institute of Saint Louis (ISL), 5 Rue du Général Cassagnou, 68301, France \\ Marion Gilson ${ }^{\S}$ and Floriane Collin ${ }^{\text {II }}$ \\ Université de Lorraine, CNRS, CRAN, F-54000 Nancy, France
}

\begin{abstract}
A vehicle's in-flight behaviour can be represented by the Newton-Euler equations of motion: usually, such a model has a nonlinear and continuous description based on ordinary differential equations. The model structure can be altered using analytic transformations and, when the model is implemented, its numerical precision may depend on the selection of a numerical solver. Hence, the selection of a model's structure and an appropriate numerical solver can become the key development issues, if the model's numerical stability, convergence, and computational complexity are to be improved. This paper assesses the influence of a model's reference frame and a numerical solver on the accuracy of calculating a projectile's trajectory, for a case study of free-flight long-range ballistic experiments. The analysis is based on two model structures: the first is a six-degree-of-freedom nonlinear description of a spin-stabilized projectile, expressed in a rolling reference frame; the second is a quasi-LPV reformulation of the same projectile model, but expressed in a non-rolling reference frame. It is shown that inappropriate selection of a numerical solver can hinder the accuracy of the nonlinear model. At the same time, the model represented in a non-rolling reference frame offers a solution with higher accuracy, better convergence properties, and significantly reduced computation time.
\end{abstract}

\section{Nomenclature}

$M$
$V$
$\alpha, \beta, \alpha_{t}$
$C_{X}, C_{N \alpha}, C_{y p \alpha}$
$C_{l p}, C_{m \alpha}, C_{m q}, C_{n p \alpha}$
$I_{x}, I_{t}$
$u, v, w$
$p, q, r$
$x_{E}, y_{E}, z_{E}$
$\phi, \theta, \psi$
$\rho$
$f_{i, \text { aero }}, m_{i, \text { aero }}$
$x, x^{\prime}$
$\mathbf{x}, \mathbf{y}$
$S$

$=$ Mach number

$=$ translational velocity, $\mathrm{m} / \mathrm{s}$

$=$ angle of attack, angle of sideslip, total angle of attack, deg

$=$ axial force, normal force slope, and Magnus force slope coefficients

$=$ roll damping, pitch moment slope, pitch damping and Magnus moment slope coefficients

$=$ longitudinal and lateral moments of inertia, $\mathrm{kg} \cdot \mathrm{m}^{2}$

$=$ linear velocities in $x, y$, and $z$ body axis, $\mathrm{m} / \mathrm{s}$

$=$ roll, pitch, and yaw rate, $\mathrm{rad} / \mathrm{s}$

$=$ center of gravity position, expressed w.r.t. Earth reference frame, $m$

$=$ Euler angles: roll, pitch, and yaw, deg

$=$ air density, $\mathrm{kg} / \mathrm{m}^{3}$

$=$ atmospheric force and moment, acting in $i$-th axis

$=$ variable in variable-roll frame, non-rolling frame

$=$ state vector, output vector

$=$ reference surface, $m^{2}$

\footnotetext{
*PhD student, Department of Aerodynamic and Exterior Ballistics, dawid.machala@isl.eu.

†Researcher, Department of Aerodynamic and Exterior Ballistics, simona.dobre@isl.eu

\$Researcher, Department of Aerodynamic and Exterior Ballistics, marie.albisser@isl.eu

§Professor, Department of Control, Identification and Diagnostics, marion.gilson@univ-lorraine.fr

II Associate professor, Department of Control, Identification and Diagnostics, floriane.collin@univ-lorraine.fr
} 


\section{Introduction}

Numerical simulations of in-flight behaviour are fundamental in the development of modern weaponry: they predict a projectile's trajectory-allowing to fulfill the safety requirements for free-flight experiments-and thus aid the assessment of its stability. The flight dynamics model, implemented for the purpose of numerical simulations, is nonlinear and depends on a set of aerodynamic coefficients that need to be quantified beforehand [1]. Multiple methods provide the means of quantifying the aerodynamic coefficients: the necessary data is typically obtained through numerical codes (Computational Fluid Dynamics), semi-empirical codes (e.g. PROjectile Design/Analysis System - PRODAS), or wind-tunnel experiments. Their results can be treated as an initial estimation of the aerodynamic coefficients, later to be used in a safety assessment of upcoming free-flight experiments, or in an identification procedure based on collected and measurement data [2, 3]. However, complex nonlinear model are problematic: the model fidelity is high, but so is the numerical complexity. Particularly, the simulation time is cumbersome. Moreover, the future utility of the model might be reduced, since the nonlinear structure may not be appropriate for a controller design.

A solution to both problems may lay in modifying the model structure. For instance, autopilots for guided projectiles are usually based on a (quasi) linear parameter-varying structure (LPV) [4] that encapsulates the nonlinearities in a quasi-linear structure, allowing the use of linear autopilot design methods [5-8]. Similarly, tasks which require multiple model runs, such as global sensitivity analysis, may benefit from an easier-to-compute representation. Recently, the authors have suggested that such a model might be obtained using a function substitution method, combined with a reference frame modification [9]. The approach exploits the established practices in quasi-LPV modelling: the function substitution [10, 11] which allows to analyze the projectile's behavior even for a non-equilibrium flight [12]; and the reference frame transformation [13, 14], which reduces the computational complexity of the system for the case of spin-stabilized ammunition. The resulting quasi-LPV model is computationally more efficient, has an identification-oriented structure, and resembles the control-oriented quasi-LPV model presented by Sève et al. [15].

However, a comparison of simulations [9] of the two models has shown non-negligible differences in their obtained state trajectories. Such differences are especially problematic in the case of long-range simulations, since the simulated results could diverge from the real trajectory over time. It is predicted that the differences between the models stem from an inappropriate selection of a numerical solver of the ordinary differential equations $(O D E)$. Indeed, while analytic derivations of the model equations in various reference frames are known for decades [13, 16, 17], the influence of $O D E$ solver selection on the accuracy of the non-rolling model's state trajectory has not been thoroughly studied.

The merit of the work lies in the evaluation of how much do the reference frame transformation and the numerical solver selection influence the accuracy of a trajectory simulation. The evaluation is performed on two models: the nonlinear one in a variable-roll reference frame, similar to these usually used in simulations for the purpose of free-flight experiments [2]; and a quasi-LPV one in a non-rolling frame, resembling these used in control [15]. In order to assess the accuracy of a numerical solution, first a trajectory considered as a reference has been found using a high order fixed-step numerical solver with high sampling frequency. Afterwards, solutions of several variable-step solvers [18] have been compared with the 'exact' solution.

The outline of the paper is as follows. In section III, the nonlinear and quasi-LPV models are described. A numerical solution, considered to be exact, is obtained in section IV through convergence and stability analysis. A comparative analysis of the models accuracy is described in section V. Finally, the paper is summarized in section VI.

\section{Mathematical model of a vehicle in flight}

The nonlinear model, based on the 6-degrees-of-freedom Newton-Euler laws of motion [19], and its representation in a quasi-LPV form [9], are shortly presented hereafter.

\section{A. Nonlinear model}

The model of the in-flight dynamics is a nonlinear state-space continuous-time structure $\mathcal{M}$ :

$$
\mathcal{M}:\left\{\begin{array}{l}
\mathbf{x}(t)=f\left(\mathbf{x}(t), \mathbf{C}\left(\mathbf{x}(t), \mathbf{p}_{a}\right)\right), \quad \mathbf{x}(0)=\mathbf{x}_{0} \\
\mathbf{y}(t)=g(\mathbf{x}(t))
\end{array}\right.
$$


where the state vector $\mathbf{x} \in \mathbb{R}^{12}$ has 12 components corresponding to the translational velocities $(u, v, w)$, rotational velocities $(p, q, r)$, position of the center of gravity of the vehicle w.r.t. Earth $\left(x_{E}, y_{E}, z_{E}\right)$, and the roll, pitch and yaw angles ( $\phi, \theta$ and $\psi$, respectively) [1]. The aerodynamic coefficients $\mathbf{C}$ are described by polynomial functions whose parameters are $\mathbf{p}_{a}$. These polynomial descriptions depend on the linear and angular velocities, as well as on the Mach number $M$ and the total angle of attack $\alpha_{t}$, which are defined by:

$$
M=\frac{V}{a} \quad \alpha_{t}=\arccos \left(\frac{u}{V}\right)
$$

where $a$ is the speed of sound, and $V$ is the total velocity, i.e. $V=\sqrt{u^{2}+v^{2}+w^{2}}$ [19]. The output vector $\mathbf{y}$ represents the measurements obtained by available sensors and observers: embedded magnetometers, accelerometers and gyroscopes, and an on-ground trajectory radar.

The full form of the nonlinear model equations in the variable-roll reference frame is described by the following set of equations, based on the Newton-Euler laws of dynamics (refer to [19]):

Translational dynamics

$$
\left[\begin{array}{c}
\dot{u} \\
\dot{v} \\
\dot{w}
\end{array}\right]=\left[\begin{array}{ccc}
0 & -r & q \\
r & 0 & -p \\
-q & p & 0
\end{array}\right]\left[\begin{array}{c}
u \\
v \\
w
\end{array}\right]+g\left[\begin{array}{c}
-s \theta \\
s \phi c \theta \\
c \phi c \theta
\end{array}\right]+1 / m\left[\begin{array}{l}
f_{x, \text { aero }} \\
f_{y, \text { aero }} \\
f_{z, \text { aero }}
\end{array}\right]
$$

Attitude dynamics

$$
\left[\begin{array}{c}
\dot{p} \\
\dot{q} \\
\dot{r}
\end{array}\right]=\left[\begin{array}{c}
0 \\
p \cdot r \frac{I_{t}-I_{x}}{I_{t}} \\
p \cdot q \frac{I_{x}-I_{z}}{I_{t}}
\end{array}\right]+\left[\begin{array}{c}
\frac{1}{I_{x}} m_{x, \text { aero }} \\
\frac{1}{I_{y}} m_{y, \text { aero }} \\
\frac{1}{I_{z}} m_{z, \text { aero }}
\end{array}\right]
$$

Translational kinematics

$$
\left[\begin{array}{c}
\dot{x}_{E} \\
\dot{y}_{E} \\
\dot{z}_{E}
\end{array}\right]\left[\begin{array}{ccc}
c \theta c \psi & s \phi s \theta c \psi-s \psi c \phi & c \phi s \theta c \psi+s \phi s \psi \\
c \theta s \psi & s \phi s \theta s \psi+c \psi c \phi & c \phi s \theta s \psi-s \phi c \psi \\
-s \theta & s \phi c \theta & c \phi c \theta
\end{array}\right]\left[\begin{array}{c}
u \\
v \\
w
\end{array}\right]
$$

Attitude kinematics

$$
\left[\begin{array}{c}
\dot{\phi} \\
\dot{\theta} \\
\dot{\psi}
\end{array}\right]=\left[\begin{array}{ccc}
1 & s \phi t \theta & c \phi t \theta \\
0 & c \phi & -s \phi \\
0 & s \phi \frac{1}{c \theta} & c \phi \frac{1}{c \theta}
\end{array}\right]\left[\begin{array}{l}
p \\
q \\
r
\end{array}\right]
$$

where the forces and moments are given by:

$$
\begin{gathered}
{\left[\begin{array}{l}
f_{x, \text { aero }} \\
f_{y, \text { aero }} \\
f_{z, \text { aero }}
\end{array}\right]=\bar{q} S\left[\begin{array}{c}
C_{X_{0}}+C_{X_{a}} s^{2} \alpha_{t} \\
-C_{y p \alpha} \frac{d}{2 V} \frac{w}{V} p-C_{N \alpha} \frac{v}{V} \\
-C_{y p \alpha} \frac{d}{2 V} \frac{v}{V} p-C_{N \alpha} \frac{w}{V}
\end{array}\right]} \\
{\left[\begin{array}{c}
m_{x, \text { aero }} \\
m_{y, \text { aero }} \\
m_{z, \text { aero }}
\end{array}\right]=\bar{q} S d\left[\begin{array}{c}
C_{l p} p \\
C_{m \alpha} \frac{w}{V}-C_{p \alpha} \frac{d}{2 V} \frac{v}{V} p+C_{m q} \frac{d}{2 V} q \\
-C_{m \alpha} \frac{v}{V}-C_{n p \alpha} \frac{d}{2 V} \frac{w}{V} p+C_{m q} \frac{d}{2 V} r
\end{array}\right] .}
\end{gathered}
$$

The state equations depend on various physical properties, measured and considered as known for each projectile, such as the mass $m$, longitudinal and lateral moments of inertia $I_{x}, I_{t}$, reference surface $S$, and diameter $d$. The dynamic pressure is denoted by $\bar{q}=\frac{1}{2} \rho V^{2}$, where $\rho$ is the air density, also assumed to be known. Symbols $s, c, t$ correspond to trigonometric sine, cosine, and tangent, respectively.

The aerodynamic coefficients can be divided into the force and moment coefficients. The force is described via the axial force $C_{X}$, the normal force slope $C_{N \alpha}$ and Magnus force slope $C_{y p \alpha}$ coefficients. Similarly, the moments are expressed through the roll damping $C_{l p}$, pitch moment coefficient slope $C_{m \alpha}$, pitch damping $C_{m q}$, and Magnus moment coefficient slope $C_{n p \alpha}$ coefficients [19]. 


\section{B. Quasi-LPV model}

In aerospace modelling, the usual choice for a coordinate system location is to rigidly fix it on the vehicle's center of mass and to subject it to all the projectile's motions [1, 14]. Alternatively, the model can be developed w.r.t. a frame that is fixed to the projectile's center of mass, but does not experience the rolling motion [14]. Such descriptions are called "variable-roll" and "non-rolling" reference frames, respectively. The latter formulation is always possible, but has an interesting property if the projectile is axis-symmetric (in $y$ and $z$ axes) and spin-stabilized: the nonlinear model equations become decoupled from its fastest changing variable, the roll angle $\phi[14,20]$. In result, the computational complexity diminishes; the non-rolling frame has been applied for this purpose in as early as in 1960s [13].

Transformation of dynamics state variables in variable-roll reference frame (3)-(4) into their non-rolling equivalents can be described using the following transformation equations [21] as:

$$
\left[\begin{array}{c}
u^{\prime} \\
v^{\prime} \\
w^{\prime}
\end{array}\right]=\left[\begin{array}{ccc}
1 & 0 & 0 \\
0 & c \phi & -s \phi \\
0 & s \phi & c \phi
\end{array}\right]\left[\begin{array}{l}
u \\
v \\
w
\end{array}\right] \quad\left[\begin{array}{l}
p^{\prime} \\
q^{\prime} \\
r^{\prime}
\end{array}\right]=\left[\begin{array}{ccc}
1 & 0 & 0 \\
0 & c \phi & -s \phi \\
0 & s \phi & c \phi
\end{array}\right]\left[\begin{array}{l}
p \\
q \\
r
\end{array}\right]
$$

where the superscript.$^{\prime}$ indicates a variable expressed w.r.t. the non-rolling frame. The kinematic state variables, i.e. Euler angles and positions are equivalent, in both frames.

A quasi-LPV model can be obtained in a non-rolling frame through a set of algebraic transformations, in accordance with the function substitution approach [10-12]. The full derivation of the model has been presented in [9], and results in the following set of equations:

$$
\dot{\mathbf{x}}^{\prime}=A\left(\mathbf{x}^{\prime}\right) \cdot \mathbf{x}^{\prime}
$$

where the non-rolling state vector $\mathbf{x}^{\prime}=\left[\begin{array}{lllllllllll}u^{\prime} & v^{\prime} & w^{\prime} & p^{\prime} & q^{\prime} & r^{\prime} & \theta^{\prime} & \psi^{\prime} & x_{E}^{\prime} & y_{E}^{\prime} & z_{E}^{\prime}\end{array}\right]^{T} \in \mathbb{R}^{11}$, and the parameterdependent state matrix can be defined as follows:

$A(x)=\left[\begin{array}{ccc}0 & -r^{\prime} & q^{\prime} \\ r^{\prime} & X_{3}\left(V, z_{E}^{\prime}\right) & r^{\prime} t \theta^{\prime}+X_{2}\left(V, z_{E}^{\prime}\right) p^{\prime} \\ -q^{\prime} & -r^{\prime} t \theta^{\prime}-X_{2}\left(V, z_{E}^{\prime}\right) p^{\prime} & X_{3}\left(V, z_{E}^{\prime}\right) \\ 0 & 0 & 0 \\ 0 & X_{7}\left(V, z_{E}^{\prime}\right) p^{\prime} & X_{5}\left(V, z_{E}^{\prime}\right) \\ 0 & -X_{5}\left(V, z_{E}^{\prime}\right) & X_{7}\left(V, z_{E}^{\prime}\right) p^{\prime} \\ 0 & 0 & 0 \\ 0 & 0 & 0 \\ c \theta c \psi & -s \psi & s \theta c \psi \\ c \theta s \psi & c \psi & s \theta s \psi \\ -s \theta & 0 & c \theta\end{array}\right.$

$X_{1}\left(u^{\prime}, V, z_{E}^{\prime}\right)-g\left(z_{E}^{\prime}\right) s \theta^{\prime}$
$p^{\prime}$
0
$\frac{g\left(z_{E}^{\prime}\right) c \theta}{p^{\prime}}$
$X_{4}\left(V, z_{E}^{\prime}\right)$
$-\frac{I_{x}}{I_{s}} r^{\prime}-\frac{r^{\prime} r^{\prime} t \theta}{p}$
$\frac{I_{x}}{I_{s}} q^{\prime}+\frac{q^{\prime} r^{\prime} t \theta}{p}$
0
0
0
0
0

0
0
0
0
$X_{6}\left(V, z_{E}^{\prime}\right)$
0
1
0
0
0
0

where:

$$
\begin{aligned}
X_{1}\left(u, V, z_{E}\right) & =-0.5 m^{-1} \rho\left(z_{E}\right) V^{2} S C_{X}(u, V) \\
X_{2}\left(V, z_{E}\right) & =0.25 m^{-1} \rho\left(z_{E}\right) S d C_{y p \alpha}(V) \\
X_{3}\left(V, z_{E}\right) & =-0.5 m^{-1} \rho\left(z_{E}\right) V S C_{N \alpha}(V) \\
X_{4}\left(V, z_{E}\right) & =0.25 \rho\left(z_{E}\right) V S d^{2} C_{l p}(V) \\
X_{5}\left(V, z_{E}\right) & =0.5 \rho\left(z_{E}\right) V S d C_{m \alpha}(V) \\
X_{6}\left(V, z_{E}\right) & =0.25 \rho\left(z_{E}\right) V S d^{2} C_{m q}(V) \\
X_{7}\left(V, z_{E}\right) & =0.25 \rho\left(z_{E}\right) S d^{2} C_{n p a}(V) .
\end{aligned}
$$

The scheduling signals of the quasi-LPV model consist of eight variables, which means that the model could be considered as a rather complex in control sense-especially that the complexity of an LPV controller increases exponentially with the number of variables [22]. However, such a structure can be deemed to be a good compromise 
between control and identification requirements: Firstly, should the complexity be reduced, fixing some of the scheduling signals will result in a control-oriented structure presented in [15]. Secondly, should the model behavior be accurate for a wide variation of flight conditions, which is necessary for the identification step [19], the model can be left 'as-is'. Out of the eight scheduling signals, five of them can be measured by available measurement techniques: the angular rates by gyroscopes, the altitude by a trajectory tracker, and the total velocity by a Doppler radar. The remaining scheduling signals, i.e. $u^{\prime}, \theta, \psi$, can be simulated or estimated, as in [23].

\section{Finding a reference solution}

Accuracy of a numerical solution to a system of $O D E$-s can be evaluated through a comparison with an analytic solution, if the latter is known. It is not usually the case for six-degrees-of-freedom Newton-Euler models of flight dynamics: for them an analytic solution can be found only by imposing additional constraints, e.g. restricting the analysis to a grid of equilibrium points, as it is done for airplane models [18, 24]. However, imposing such a restriction would be impractical since the end goal of the project is to develop an identification-oriented model.

An alternative approach, used in the present work, consists of applying a numerical solver with-impractically strict-accuracy constraints; considering its solution as a reference; and using the reference in order to evaluate if numerical solvers with less strict constraints provide a comparable accuracy.

Numerical solvers consist of fixed- and variable-step categories. The latter is applicable to stiff problems, has a shorter computation time and better convergence properties [25], for which reasons it is embedded in Matlab [18]. The fixed-step category, on the other hand, can be slower in solving an $O D E$ due to inability to modify the time-step, albeit offer a direct control over the solver's order and sampling time, allowing to easily observe convergence of a solution. Hence, in order to find a reference solution, it is decided to apply a fixed-step $O D E$ solver with a high order and high sampling frequency. Such a solution can be then used in assessment of much faster variable-step solvers, so that the most accurate solver can be selected. The approach is visualized in the ideogram in Fig. 1

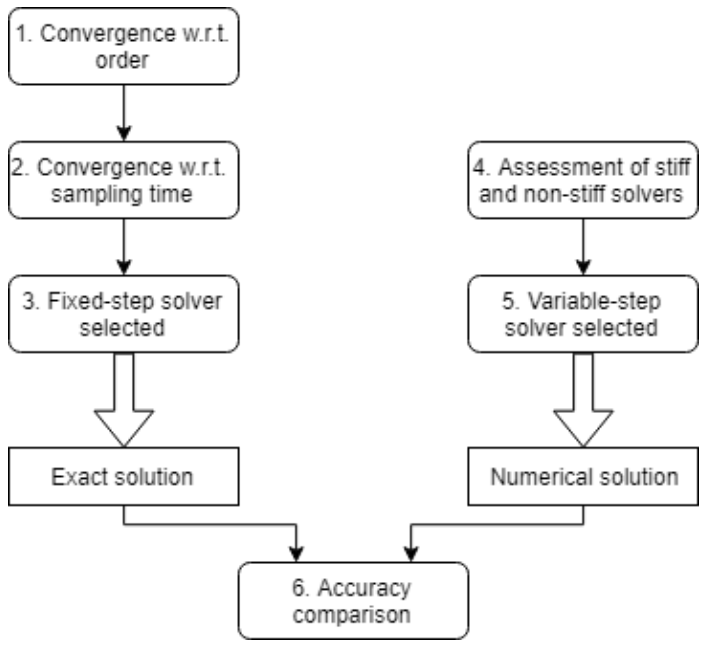

Fig. 1 Comparison of exact and numerical solutions

It is important to assess if solutions converge and are stable, prior to declaring one of them as a reference solution. They can be evaluated by varying the solver's order and sampling time. Herein, it is proposed to find an 'exact' (as in Fig. 1) solution using the following approach:

1) Obtain a number of numerical solutions using fixed-step solver for a range of solver's orders and sampling times, in such a way, that the solutions are expected to be stable and accurate, e.g. by arbitrarily specifying the maximum step size [26, 27].

2) Test if the solutions converge with an increasing solver order.

3) Test if the solutions converge with an increasing sampling.

4) If the solutions are stable and converging, select the solution obtained with the highest order and highest sampling time, as the 'exact' one.

Such a procedure allows to find a solution that can be considered as a reference, albeit is heavily computationally 
expensive. This computational cost motivates the search for a much faster variable-step solver that produces the most similar results.

Using the procedure, both the nonlinear and quasi-LPV models have been analyzed. The test scenario applied in the analysis corresponds to a parabolic flight trajectory. The set of initial values is as follows: $u_{0}=700 \mathrm{~m} / \mathrm{s}, \mathrm{p}_{0}=1420$ $\mathrm{rad} / \mathrm{s}, \theta_{0}=15 \mathrm{deg}, \psi_{0}=0 \mathrm{deg}$. The remainder initial state variables are set to zero. Such an initial elevation angle and velocity would result in a flight lasting approximately $30 \mathrm{~s}$ in real life.

\section{A. Solution of the nonlinear model}

Firstly, the influence of the solver's order is investigated. The sampling time has been fixed to $20 \mu s$ : the selection is motivated by the highest frequency of the model i.e. the roll angle evolution, which has a period of approximately 5 $m s$ - it is of two orders of magnitude larger than the sampling time, allowing to obtain an accurate solution. Fixed-step solvers with orders ranging from one to five have been applied.

An exemplary trajectory of a pitch rate $q$ evolution over time has been visualized in Fig. 2. It can be observed that for each increase in solver's order (trajectories noted as ode2-ode5, ode 1 is not shown, since the solution was unstable) the difference between the solutions obtained for the given order and the highest tested order is decreasing. The solution calculated for the fifth order solver (i.e. Runge-Kutta method) differ only by approximately $2 e-8$ from the fourth order solution, i.e. the magnitude of the difference is $10^{8}$ smaller than the magnitude of the pitch rate signal. The convergence of solutions is even more visible when analyzing the root mean square $(R M S)$ differences, as presented in Fig. 3 a) for three exemplary state variables: $y, \theta$, and $\psi$. For the analyzed flight scenario, these variables reach values from the range of $y \in 0-120 \mathrm{~m}, \theta \in 0-22 \mathrm{deg}$, and $\psi \in \pm 30 \mathrm{deg}$. The magnitude of $R M S$ differences between the reference solution and the lower order solutions is decreasing ten- to thousandfold with each increase in solver's order. Therefore, with an increase in order, the solution gets more stable and converges more easily. Moreover, the solution for the fifth order differs only negligibly from the fourth order solution, hence the fifth order can be considered as a sufficiently accurate.
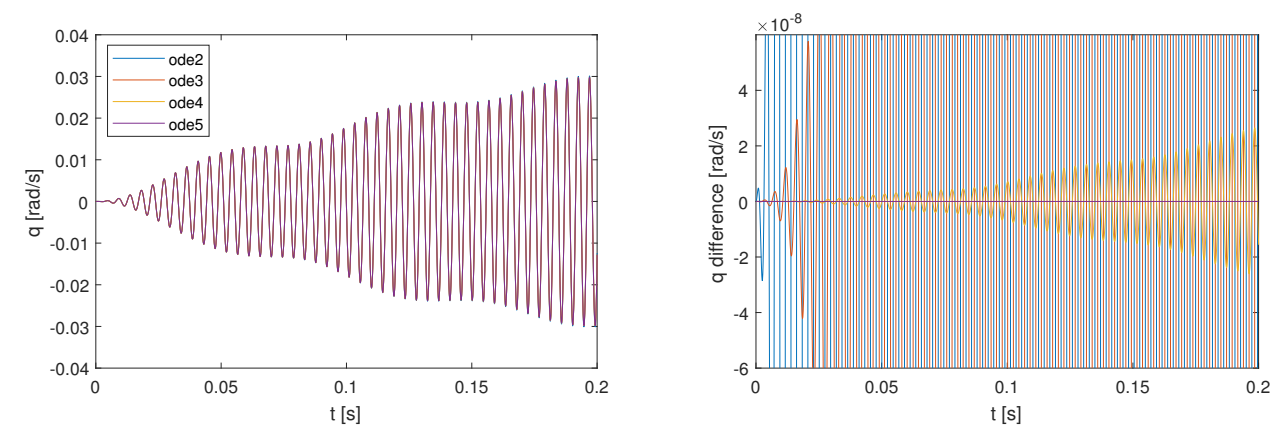

Fig. 2 The first $0.2 s$ of the pitch rate $q$ evolution (left): convergence with increasing solver's order (right)

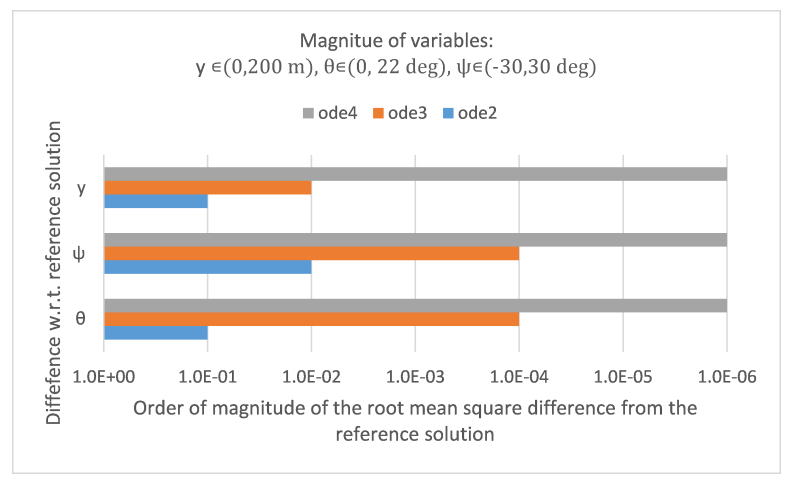

(a) Increase in accuracy w.r.t. increasing $O D E$ order

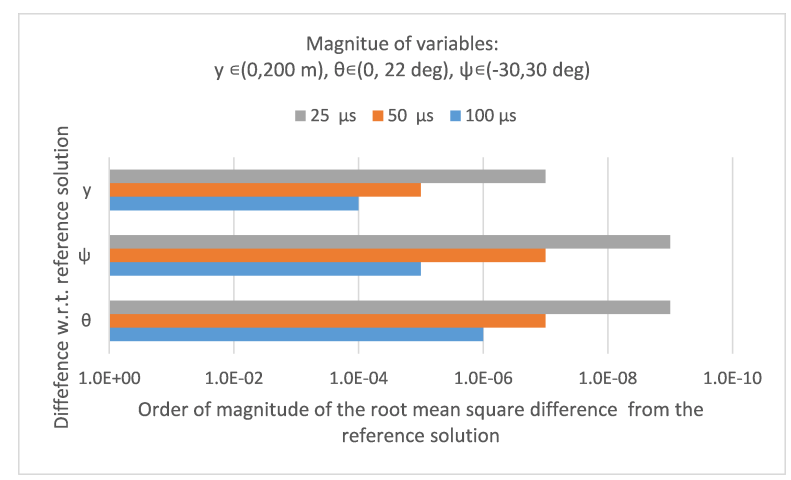

(b) Increase in accuracy w.r.t. decreasing time step

Fig. 3 Root mean square difference w.r.t. solution of the fifth order solver with $20 \mu s$ : convergence with increasing solver's order and sampling time 
Secondly, the influence of the sampling time has been investigated. A solver's order equal to five, considered in the prequel as sufficiently accurate, has been used for all the tests. The sampling time ranged from 20 to $100 \mu s$. The simulation results are summarized in Fig. $3 \mathrm{~b}$ ). The $R M S$ difference between simulations decreases ten- to hundredfold for a twofold increase in sampling frequency, hence the solutions are considered to be converging for a decreasing sampling time.

Therefore, the solutions converge and become more stable w.r.t. the solver's order and sampling time, while the differences between solutions become negligibly small. Hence, the solution obtained for the highest order and the most dense sampling, i.e. the fifth order solution for $20 \mu$ s sampling is considered as a reference for the future analysis.

\section{B. Solution of the quasi-LPV model}

Numerical analysis of the quasi-LPV model in the non-rolling frame has been performed using the same procedure as for the nonlinear model in the variable-roll reference frame. Due to slower system's dynamics (the equations are decoupled from the fast-varying roll angle [14]), the sampling time of $300 \mu s$ has been sufficient to obtain a stable solution, even for the first-order solver.

The exemplary results showing the influence of the solver's order are presented in Fig. 4. The $R M S$ of the difference decreases thousandfold with each increase in solver's order, and the differences are smaller than for the case of variable-roll model. Such effect stems from slower system dynamics. It can also be observed for the example of the pitch rate $q$ in Fig. 5 , whose variations are slower than in Fig. 2.

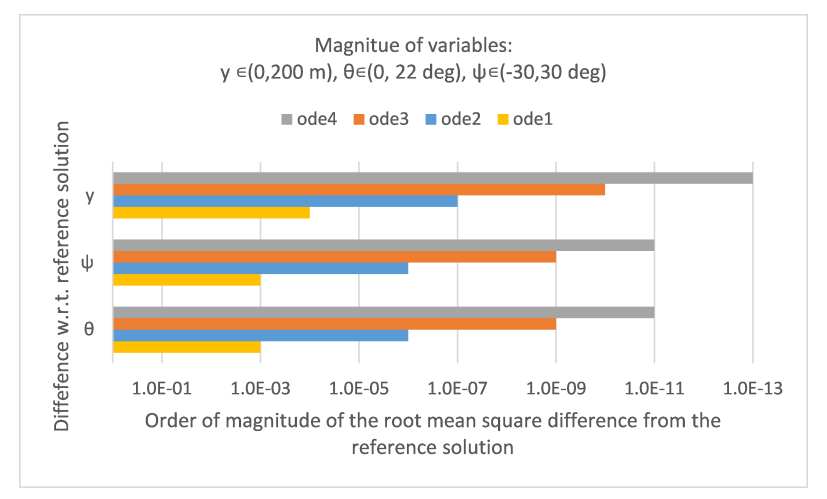

Fig. 4 Root mean square difference w.r.t. solution of the fifth order solver with $300 \mu s$ : the solutions converge with increasing solver's order

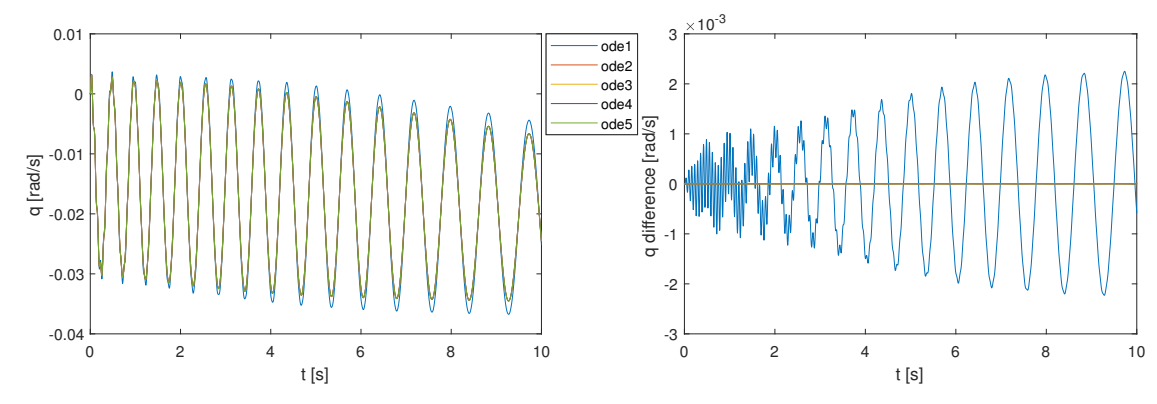

Fig. 5 Convergence of a numerical solution - pitch rate $q$ w.r.t. increasing solver's order

\section{Summary}

Using fixed-step solvers, stability and convergence of solutions of the two models has been assessed and their reference solutions have been obtained. Additionally, it can be observed that for the same initial conditions and solver's order, the non-rolling frame results are of higher accuracy than the variable-roll ones. 
The duration of a simulation is significantly reduced by the change of model's structure, as can be seen in Table 1 The fixed-step simulation lasts approximately 50 minutes for the nonlinear model, and approximately 3 minutes for the quasi-LPV model. The difference in time stems from different sampling used in each $O D E$ settings.

\begin{tabular}{l|l} 
Model structure & Computation time \\
\hline Nonlinear & 50 minutes \\
Quasi-LPV & 3 minutes
\end{tabular}

Table 1 Duration of a simulation: model structure influences the computation time

\section{Assessment of the variable-step solvers}

In the previous section, a numerical solution considered as a reference has been found for both models. However, the reference solutions are computationally expensive to obtain due to strict order and sampling time constraints. In order to reduce the computational complexity, the variable-step solvers are analyzed in this section: their solutions are easier to compute due to varying time step. Their accuracy is assessed w.r.t. the reference solutions, as shown in Fig. 1

The variable-step $O D E$ solvers available in Matlab, including the ones adapted for non-stiff problems like the Runge-Kutta based ode 23 and ode 45 or the Adams-Bashforth-Moulton based ode113, and the ones adapted for stiff problems, such as the variable-step-variable-order ode $15 s[18]$ are used in the following tests.

\section{A. Nonlinear model analysis}

The reference solution to the nonlinear model obtained in the previous section allows to assess accuracy of the variable-step solvers. The same set of initial state values is used.

As can be seen based on the results summary presented in Table 2 , the selection of a solver influences the accuracy and computation time significantly. The solver adapted to stiff problems, ode $15 \mathrm{~s}$, achieves the lowest accuracy of all the solvers in the case of kinematic variables, such as position $y$ and attitude $\theta, \psi$. Over a long-distance simulation, such discrepancy can have a significant effect on the accuracy of predicted trajectory. On the other hand, the ode45 solver has allowed to obtain the highest accuracy for all the state variables, while requiring less than 6 minutes (as compared to 50 minutes of calculating the reference solution) to compute the trajectory.

\begin{tabular}{|c|c|c|c|c|c|c|}
\hline \multirow{2}{*}{ Solver } & Computation & \multicolumn{5}{|c|}{ Root mean square error $w . r . t$. the reference solution } \\
\cline { 3 - 7 } & time & $\mathrm{u}[\mathrm{m} / \mathrm{s}]$ & $\mathrm{q}[\mathrm{rad} / \mathrm{s}]$ & $\theta[\mathrm{deg}]$ & $\psi[\mathrm{deg}]$ & $\mathrm{y}[\mathrm{m}]$ \\
\hline ode 45 & $5 \min 34 \mathrm{~s}$ & $\mathrm{e}-04$ & $\mathrm{e}-04$ & $\mathrm{e}-05$ & $\mathrm{e}-05$ & $\mathrm{e}-03$ \\
ode 23 & $20 \mathrm{~min} 37 \mathrm{~s}$ & $\mathrm{e}-04$ & $\mathrm{e}-04$ & $\mathrm{e}-04$ & $\mathrm{e}-04$ & $\mathrm{e}-02$ \\
ode 113 & $1 \mathrm{~min} 35 \mathrm{~s}$ & $\mathrm{e}-04$ & $\mathrm{e}-04$ & $\mathrm{e}-04$ & $\mathrm{e}-04$ & $\mathrm{e}-03$ \\
ode $15 s$ & $1 \min 49 \mathrm{~s}$ & $\mathrm{e}-03$ & $\mathrm{e}-04$ & $\mathrm{e}-03$ & $\mathrm{e}-03$ & $\mathrm{e}-01$ \\
\hline
\end{tabular}

Table 2 Variable-roll reference frame: order of magnitude of the $R M S$ difference between the reference solution and variable-step simulations' results of the nonlinear model

\section{B. Quasi-LPV model analysis}

The reference solution to the quasi-LPV model in the non-rolling frame obtained in the previous section allows to assess accuracy of the variable-step solutions.

Similarly as in the previous case, the variable-step solvers available in Matlab have been tested with the results summarized in Table 3. Since the roll angle is decoupled, the problem is less stiff and less computationally expensive. It can be observed that all the variable-step solvers have similar accuracy, and all the solvers offer higher accuracy and shorter computation time than in the case of the nonlinear model. Consequently, it can be argued that the model represented in a non-rolling reference frame is numerically more stable. Among the tested solvers, ode 45 and ode $15 s$ have the lowest computation time. Therefore, it is recommended to use them in the non-rolling frame simulations. The simulation lasts 4 to 5 seconds, which is shorter than the duration of the flight in real world. 


\begin{tabular}{|c|c|c|c|c|c|c|}
\hline \multirow{2}{*}{ Solver } & Computation & \multicolumn{5}{|c|}{ Root mean square error w.r.t. the reference solution } \\
\cline { 3 - 7 } & time & $\mathrm{u}[\mathrm{m} / \mathrm{s}]$ & $\mathrm{q}[\mathrm{rad} / \mathrm{s}]$ & $\theta[\mathrm{deg}]$ & $\psi[\mathrm{deg}]$ & $\mathrm{y}[\mathrm{m}]$ \\
\hline ode 45 & $5 \mathrm{~s}$ & $\mathrm{e}-04$ & $\mathrm{e}-06$ & $\mathrm{e}-05$ & $\mathrm{e}-05$ & $\mathrm{e}-05$ \\
ode 23 & $9 \mathrm{~s}$ & $\mathrm{e}-04$ & $\mathrm{e}-06$ & $\mathrm{e}-05$ & $\mathrm{e}-05$ & $\mathrm{e}-05$ \\
ode 113 & $8 \mathrm{~s}$ & $\mathrm{e}-04$ & $\mathrm{e}-06$ & $\mathrm{e}-05$ & $\mathrm{e}-05$ & $\mathrm{e}-05$ \\
ode $15 s$ & $4 \mathrm{~s}$ & $\mathrm{e}-04$ & $\mathrm{e}-06$ & $\mathrm{e}-05$ & $\mathrm{e}-05$ & $\mathrm{e}-05$ \\
\hline
\end{tabular}

Table 3 Non-rolling reference frame: order of magnitude of the $R M S$ difference between the reference solution and variable-step simulations' results of the quasi-LPV model

\section{Summary}

Using variable-step solvers embedded in Matlab the numerical solutions have been obtained for both the nonlinear and quasi-LPV models. As predicted, the approach is less computationally expensive than using fixed-step solver, albeit for the price of being more errors-prone. Based on the results presented in Tables 2 and 3 , it is concluded that the most accurate $O D E$ solver is $o d e 45$, which for both models allows to obtain a solution similar to the reference one.

\section{Comparative analysis of the models' trajectories}

The previous section shows how to obtain accurate solutions using the fixed-step and variable-step solvers for both reference frames. The analytic derivation presented in [13, 16] suggests that the variable-roll and non-rolling reference frame can be considered as equivalent to each other: the results obtained in either of the frames can be converted to the other one using the transformation matrices 9 ) or their inverse. In order to test these premises on numerical grounds, an additional long-range simulation has been performed.

The non-zero initial conditions correspond to $u_{0}=798.4 \mathrm{~m} / \mathrm{s}, p_{0}=1619 \mathrm{rad} / \mathrm{s}, \theta_{0}=63 \mathrm{deg}$, and $\psi_{0}=-0.046$ deg. Resulting parabolic flight lasts approximately 100 seconds: numerical discrepancies should become visible over such a long trajectory.

Firstly, to emphasize the importance of the selection of an appropriate $O D E$ solver, a variable-step solution has been calculated for both models using ode15s: such a selection could be made based on the suspected stiff nature of the nonlinear model. However, as seen in Table 2, it is the least accurate solver in practice. The results of such a simulation are presented on an example of the total angle of attack evolution in Fig. 6 Indeed, the difference between reference frames reaches up to one degree at the end of the flight, which is unacceptably high.
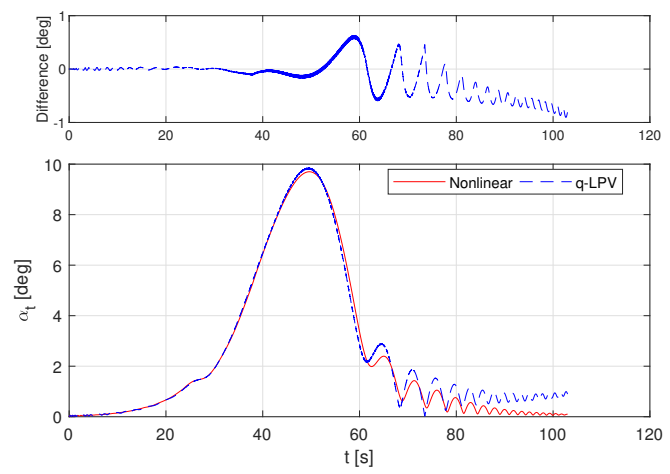

Fig. 6 Consequences of poor selection of the $O D E$ solvers: inaccurate long-range $\alpha_{t}$ trajectory

However, the difference can be reduced, using the procedure described in the two previous sections. A reference solution for each frame is found using the fifth-order fixed-step $O D E$ solver, with the sampling time equal to $20 \mu s$. Two exemplary variables, the total angle of attack and the total velocity, are plotted in Fig. 7 for comparison, as they should be equal in both frames. Indeed, the maximum difference between the reference solutions for both frames is lower than $2 \mu \mathrm{deg}$ and $1 \mu \mathrm{m} / \mathrm{s}$, respectively, which is more accurate than available measurement techniques. Hence, the reference solutions are equivalent, as predicted by analytic developments. 


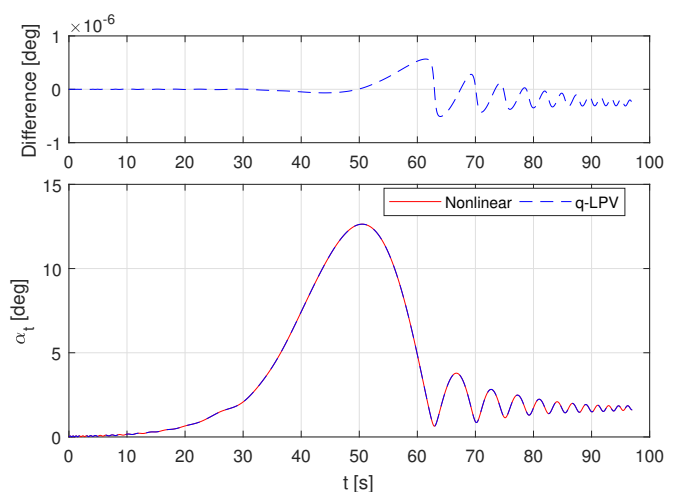

(a) Total angle of attack
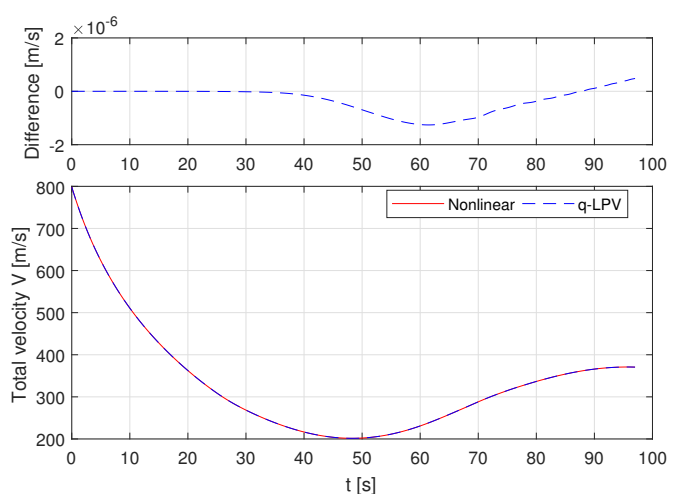

(b) Total velocity

Fig. 7 Comparison of fixed-step solutions in both frames

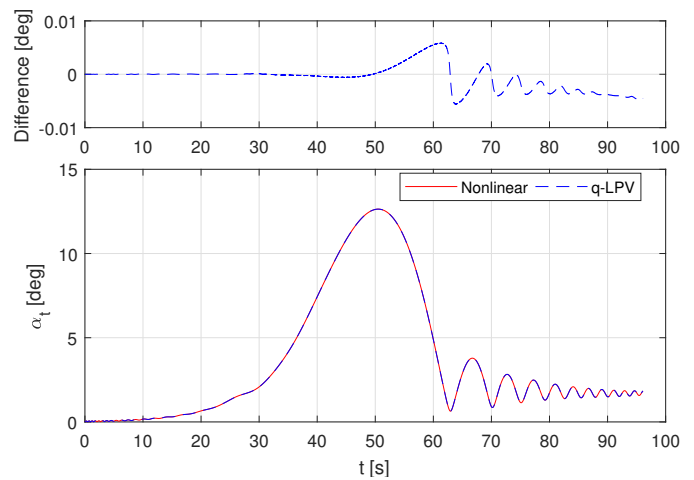

(a) Total angle of attack
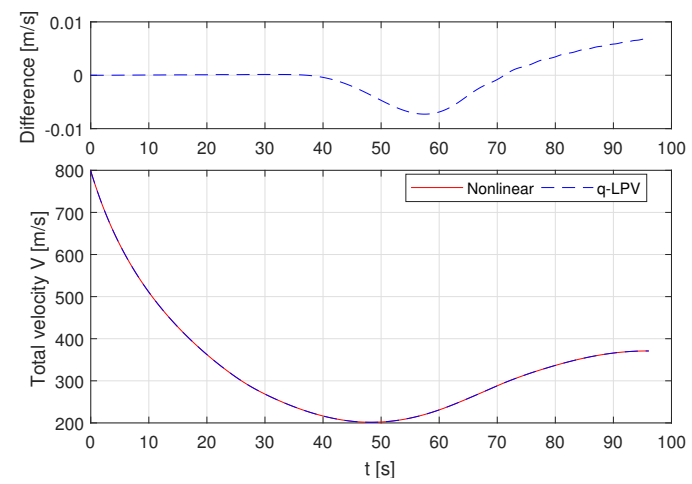

(b) Total velocity

Fig. 8 Comparison of variable-step solutions in both frames

In the next step, the variable-step solvers have been applied. Accordingly with the results presented in Table 2 and 3 , solver ode 45 has been used. The resulting trajectories of the total angle of attack and the total velocity are presented in Fig. 8. The maximum difference between the variable-step solvers remains smaller than $0.01 \mathrm{deg}$ and $0.01 \mathrm{~m} / \mathrm{s}$, respectively. Such a difference, albeit higher than in the case of fixed-step simulations, can still be considered acceptable compared to magnitude of the signals.

Finally, the final-step error for selected state variables, calculated w.r.t. the reference solution for each solver, is presented in Table 4. If compared, the state variables obtained using fixed-step solver differ in the range of orders of magnitude between $e-04$ and $e-07$; which corresponds to the accuracy presented in Fig. 3 Should a higher accuracy be required, simulation with $O D E$ of orders six or above would have to be used.

Additionally, it can be seen that accuracy of the simulation obtained with variable-step solvers is significantly higher for the case of the non-rolling reference frame: when compared to the variable-roll frame simulation, the state variables are at least 100 times more accurate. It can be concluded that simulating the trajectory in the non-rolling frame first, and transforming the results to the variable-roll frame afterwards, is in fact both faster and more accurate than simulating them only in the rolling frame.

Consequently, the non-rolling frame can be considered as an accurate alternative for the rolling-frame simulations. The benefits are the higher robustness to numerical solver errors and faster computation. 


\begin{tabular}{|c|c|c|c|c|c|}
\hline \multirow{2}{*}{ State } & \multirow{2}{*}{ Unit } & \multirow{2}{*}{ Range } & \multicolumn{3}{|c|}{ Final step difference w.r.t. the reference solution: } \\
\cline { 4 - 6 } & & & fixed-step non-rolling & variable-step variable-roll & variable-step non-rolling \\
\hline$u$ & $\mathrm{~m} / \mathrm{s}$ & $400-800$ & $\mathrm{e}-07$ & $\mathrm{e}-03$ & $\mathrm{e}-06$ \\
$p$ & $\mathrm{rad} / \mathrm{s}$ & $1100-1700$ & $\mathrm{e}-07$ & $\mathrm{e}-03$ & $\mathrm{e}-06$ \\
$\phi$ & $\mathrm{deg}$ & $0-360$ & $\mathrm{e}-04$ & $\mathrm{e}-01$ & $\mathrm{e}-04$ \\
$\theta$ & $\mathrm{deg}$ & \pm 70 & $\mathrm{e}-07$ & $\mathrm{e}-04$ & $\mathrm{e}-08$ \\
$\psi$ & $\mathrm{deg}$ & $0-16$ & $\mathrm{e}-06$ & $\mathrm{e}-04$ & $\mathrm{e}-07$ \\
$x_{E}$ & $\mathrm{~m}$ & $0-20000$ & $\mathrm{e}-05$ & 1.02 & $\mathrm{e}-05$ \\
$y_{E}$ & $\mathrm{~m}$ & $0-1000$ & $\mathrm{e}-04$ & -3.75 & $\mathrm{e}-06$ \\
$z_{E}$ & $\mathrm{~m}$ & $0-12000$ & $\mathrm{e}-05$ & -0.61 & $\mathrm{e}-05$ \\
\hline
\end{tabular}

Table 4 Range of variation of state variables and the order of magnitude of the final-step difference w.r.t. the reference solution

\section{Conclusion}

In the paper, two models of vehicle's in-flight behavior have been presented: the nonlinear, developed in a variable-roll reference frame, and a proposed identification-oriented quasi-LPV, developed in a non-rolling reference frame. Their numerical accuracy has been studied, principally w.r.t. the selection of an ordinary differential equations solver.

Both models were tested through simulations, with initial conditions corresponding to these of real-world long-range ballistic experiments. A numerical solution close to the exact one has been obtained by applying a fixed-step numerical solver with a high order and high sampling frequency. Such a method is computationally expensive but allows to obtain accurate results. Afterwards, the much faster variable step solvers have been applied. Their accuracy has been analyzed w.r.t. the fixed-step solver's solution, considered as a reference.

It is shown that while it is possible to obtain a numerical solution for both representations, the quasi-LPV model in the non-rolling reference frames offers a solution with higher accuracy, better convergence properties, and shorter computation time. As such, it will be used in the future analyses, aiming to perform a global sensitivity of the system.

\section{Acknowledgments}

This work is in part financed by the French Defense Procurement Agency (DGA, Direction Générale de l'Armement).

\section{References}

[1] Zipfel, P. H., Modeling and Simulation of Aerospace Vehicle Dynamics, $2^{\text {nd }}$ ed., AIAA Inc., Blacksburg, 2007.

[2] Albisser, M., Dobre, S., Berner, C., Thomassin, M., and Garnier, H., "Aerodynamic Coefficient Identification of a Space Vehicle from Multiple Free-Flight Tests,” Journal of Spacecraft and Rockets, Vol. 54, No. 2, 2017, pp. 426-435.

[3] Vitale, A., and Corraro, F., "Identification from flight data of the italian unmanned space vehicle," 16th IFAC Symp. on Sys. Iden., Vol. 45, 2012.

[4] Tóth, R., Modeling and Identification of Linear Parameter-Varying Systems, Vol. 403, Springer, 2010. doi:10.1007/978-3-64213812-6.

[5] Al-jiboory, A., Zhu, G. G., Swei, S. S.-M., Su, W., and Nguyen, N. T., "LPV Model Development for a Flexible Wing Aircraft," 58th AIAA/ASCE/AHS/ASC Structures, Structural Dynamics, and Materials Conference, 2017.

[6] Fleischmann, S., Theodoulis, S., Laroche, E., Wallner, E., and Harcaut, J.-P., “A Systematic LPV/LFR Modelling Approach Optimized for Linearised Gain Scheduling Control Synthesis," AIAA Modeling and Simulation Technologies Conference, 2016.

[7] Berger, T., Tischler, M. B., Hagerott, S. G., Cotting, M. C., Gray, W. R., Gresham, J., George, J., Krogh, K., D’Argenio, A., and Howland, J., "Development and Validation of a Flight-Identified Full-Envelope Business Jet Simulation Model Using a Stitching Architecture," AIAA Modeling and Simulation Technologies Conference, 2017.

[8] Rugh, W. J., and Shamma, J. S., "Research on gain scheduling," Automatica, Vol. 36, No. 10, 2000, pp. 1401-1425. doi:10.1016/S0005-1098(00)00058-3. 
[9] Machala, D., Dobre, S., Albisser, M., Collin, F., and Gilson, M., 18th IFAC Symposium on System Identification, SYSID 2018 , 2018.

[10] Tan, W., Packard, A. K., and Balas, G. J., "Quasi-LPV Modeling and LPV Control of a Generic Missile,” Proceeding of the American Control Conference, 2000.

[11] Pfifer, H., "Quasi-LPV Model of a NDI-Controlled Missile Based on Function Substitution,” AIAA GNC Conference, 2012. doi:10.2514/6.2012-4970.

[12] Marcos, A. E., and Balas, G., "Linear parameter varying modeling of the Boeing 747-100/200 longitudinal motion," AIAA GNC Conference, 2001.

[13] Barnett, B., “Trajectory equations for a six-degree-of-freedom missile,” Tech. rep., Picatinny Arsenal, 1962.

[14] Wernert, P., Theodoulis, S., and Morel, Y., "Flight Dynamics Properties of 155 mm Spin-Stabilized Projectiles Analyzed in Different Body Frames,” AIAA AFM Conference, 2010. doi:10.2514/6.2010-7640.

[15] Sève, F., Theodoulis, S., Wernert, P., Zasadzinski, M., and Boutayeb, M., "Pitch/Yaw Channels Control Design for a 155mm Projectile with Rotating Canards, using a H-infinity Loop-Shaping Design Procedure," AIAA GNC Conference, 2014. doi:10.2514/6.2014-1474.

[16] Barnett, B., "Trajectory equations for a six-degree-of-freedom missile using a fixed-plane coordinate system," Tech. rep., Picatinny Arsenal, Dover, New Jersey, 1966.

[17] Whyte, R. H., and Hathaway, W. H., “Aeroballistic Range Data Reduction Technique Using Numerical Integration,” Tech. rep., 1974.

[18] Shampine, L. F., and Reichelt, M. W., “The MATLAB ODE Suite,” SIAM Journal on Scientific Computing, Vol. 18, No. 1, 1997, pp. 1-22. doi:10.1137/S1064827594276424, URL http://epubs.siam.org/doi/10.1137/S1064827594276424

[19] Albisser, M., "Identification of aerodynamic coefficients from free flight data," Ph.D. thesis, University of Lorraine, 2015.

[20] Wernert, P., "Simulations de trajectoires de projectiles à 6 degrés de liberté dans le cadre de la mécanique du vol avion [Simulations of six-degrees-of-freedom projectile trajectories in the framework of the aircraft flight mechanics].” Tech. rep., French-German Research Institute of Saint-Louis (ISL), 2007.

[21] Fischer, M., and Hathaway, W., “Aeroballistic Research Facility Data Analysis System,” Tech. rep., Eglin Air Force Base, USA, 1988.

[22] Kwiatkowski, A., and Werner, H., "Parameter Reduction for LPV Systems via Principal Components Analysis," IFAC Proceedings Volumes, Vol. 38, No. 1, 2005, pp. 596-601.

[23] Changey, S., Pecheur, E., and Brunner, T., "Attitude Estimation of a projectile using Magnetometers and Accelerometers: Experimental Validation,” IEEE/ION PLANS Symposium 2014, 2014, pp. 1168-1173.

[24] Cook, M., Flight Dynamics Principles, $2^{\text {nd }}$ ed., Elsevier, 2007.

[25] Ashino, R., Nagase, M., and Vaillancourt, R., "Behind and Beyond the MATLAB ODE Suite," Computers and mathematics with applications, Vol. 40, 2000, pp. 491-512. doi:10.1016/S0898-1221(00)00175-9.

[26] Holsapple, R., Iyer, R., and Doman, D., "Variable Step-size Selection Methods for Implicit Integration Schemes for ODES," International Journal of Numerical Analysis and Modeling, Vol. 4, No. 2, 2007, pp. 210-240. doi:10.1109/ACC.2006.1657179.

[27] Hull, T. E., Enright, W. H., Fellen, B. M., and Sedgwick, A. E., "Comparing Numerical Methods for Ordinary Differential Equations," SIAM Journal on Numerical Analysis, Vol. 9, No. 4, 1974, pp. 603-637. doi:10.1137/0711054, URL http: //epubs.siam.org/doi/10.1137/0711054 